\title{
Transcatheter Closure of a Mitral Valve Paravalvular Leak in an Infant
}

\author{
Ugonna Nwankwo, MD*, James Goldsmith, MD, Sara Trucco, MD, Jacqueline Kreutzer, MD \\ Department of Cardiology, Children's Hospital of Pittsburgh of UPMC, Pittsburgh, Pennsylvania, USA
}

\begin{abstract}
Mitral valve replacement, which is a relatively rare procedure in young children, can be complicated by paravalvular leaks (PVLs). Here, we report the first successful percutaneous treatment of a PVL in an infant. The patient was a 7-month-old, 6 kg boy with a history of Shone's complex after aortic arch reconstruction and balloon valvuloplasty of the mitral valve. Due to severe mitral insufficiency, he underwent mitral valve replacement with a 16-mm Medtronic open-pivot AP 360 mechanical aortic valve in the supra-annular mitral position at 5 months of age. He developed a posterior PVL post-operatively with persistent hemolysis requiring repeated transfusions. Medical therapy with pentoxifylline failed. Thus, 2 months post-operatively, he was referred for percutaneous cardiac catheterization for PVL closure. Transesophageal echocardiography (TEE) identified a small PVL between the mouth of the left atrial appendage and the left upper pulmonary vein. A trans-septal approach was utilized. Using a Cobra catheter, a 0.018" floppy wire was advanced from the venous sheath through the leak and into the ascending aorta, where it was snared by a multisnare introduced from the arterial sheath. A 4-mm Amplatzer vascular plug II was then positioned anterogradely and successfully deployed in the tract. No significant leak was visualized by angiography or TEE, and the child's hemolysis subsided. Our case demonstrates that device closure of mitral valve PVL in a very small child is technically feasible and may obviate repeat surgery.
\end{abstract}

\section{Key Words}

Catheterization - Paravalvular Prosthetic $\cdot$ Mitral valve $\cdot$ Pediatric

regurgitation •

(c) 2017 Journal of Structural Heart Disease Published by Science International Corp. ISSN 2326-4004

Fax +1 2037853346

E-Mail: jshd@scienceinternational.org

http://structuralheartdisease.org/

\section{Introduction}

Paravalvular prosthetic valve leak (PVL) is a potentially significant complication of surgical valve replacement, with a reported incidence of up to $18 \%$ for aortic valves and $23 \%$ for mitral valves in adult patients [1]. Many factors can contribute to PVL due to an incomplete seal between the prosthetic ring and native valve annulus, including annular calcification, infection, suboptimal surgical technique, suboptimal prosthesis sizing, or any cause of tissue friability [2]. Although a small PVL is often asymptomatic, it can lead to significant hemolysis, whereas moderate or large PVLs can manifest with signs and symptoms secondary to increased hemodynamic burden, including pulmonary hypertension and congestive heart failure [3]. In young children, mitral valve replacement is a relatively rare procedure, and there are limited data on PVL intervention in children under 12 months old. To our knowledge, this is the first reported case of transcatheter paravalvular leak closure in a child under 12 months of age.

\section{Case Presentation}

Appropriate signed consent was obtained. The patient was a 7-month-old, 6 kg boy born with Shone's variant consisting of coarctation of the aorta, bicuspid aortic valve, and congenital mitral valve stenosis. On the first day of life, he was taken to the operating room for coarctectomy and aortic arch reconstruction. He initially did well but developed progressive mitral stenosis with associated respiratory distress, feeding intolerance, and failure to thrive, for which he

* Corresponding author:

Ugonna Nwankwo, MD

Sibley Heart Center Cardiology

Children's Healthcare of Atlanta

1405 Clifton Rd NE, Atlanta, GA 30322, USA

Tel.: +1 336391 6621; Fax: + 1770488 9431; E-Mail: nwankwou@kidsheart.com 
subsequently underwent cardiac catheterization at 4 months of age. During catheterization, he was found to have severe mitral stenosis with a mitral valve gradient by planimetry (mean gradient of $13-16 \mathrm{mmHg}$ ), a capillary wedge pressure to left ventricular end-diastolic pressure gradient of $16 \mathrm{mmHg}$, as well as secondary pulmonary hypertension with a pulmonary vascular resistance of 7.1 indexed Wood units that was poorly responsive to vasodilator therapy. Balloon valvuloplasty of the mitral valve was performed to alleviate left atrial hypertension, but there was negligible improvement in the valve gradient, and the patient developed severe mitral insufficiency post-valvuloplasty. He had a prolonged post-catheterization hospitalization with difficulty weaning from mechanical ventilation as well as recurrent respiratory distress and feeding intolerance recalcitrant to medical management. As his anatomy was deemed not amenable to surgical repair, he underwent mitral valve replacement with a 16-mm Medtronic open-pivot AP 360 mechanical aortic valve in the supra-annular mitral position at 5 months of age. His post-operative course was complicated by a small PVL first noted upon post-operative transesophageal echocardiography (TEE). In addition, he developed a moderate pericardial effusion that eventually required surgical intervention with a pericardial window. The PVL was noted by all subsequent TEE and was localized to the posterolateral ring of the valve. There was no worsening of the leak upon serial imaging; however, the patient was readmitted for an elevated international normalized ratio and was found to have developed persistent hemolysis with elevated plasma-free hemoglobin and lactase dehydrogenase levels, requiring repeated blood transfusions. Attempted medical therapy with pentoxifylline failed, thus 2 months post-operatively, the patient was referred to the cardiac catheterization laboratory for percutaneous PVL closure.

Hemodynamic findings are summarized in Table 1. The most significant findings were elevated left atrial pressure, elevated mitral valve peak gradient, and elevated left ventricular end-diastolic pressure suggestive of diastolic dysfunction. Procedural TEE identified a small-to-moderate PVL between the mouth of the left atrial appendage and the left upper pulmonary vein measuring $1.2 \mathrm{~mm}$, which was confirmed by an-
Table 1. Hemodynamic findings before and after paravalvular leak closure.

\begin{tabular}{lcc}
\hline Site & $\begin{array}{c}\text { Before Closure } \\
\text { (in mm Hg) }\end{array}$ & $\begin{array}{c}\text { After Closure } \\
\text { (in mm Hg) }\end{array}$ \\
\hline RA & $5 / 7 / 4$ & $8 / 10 / 7$ \\
RV & $42 / 3$ & - \\
MPA & $32 / 14 / 23$ & - \\
RPA & $30 / 11 / 21$ & - \\
RPAW & $17 / 16 / 14$ & - \\
LPA & $35 / 15 / 25$ & - \\
LPAW & $17 / 12 / 17$ & - \\
LA & $20 / 18 / 14$ & $15 / 20 / 13$ \\
LV & $88 / 14$ & $98 / 12$ \\
AAO & $82 / 38 / 57$ & $90 / 40 / 62$ \\
DAO & $71 / 38 / 53$ & $71 / 39 / 54$ \\
\hline
\end{tabular}

$\mathrm{AAO}=$ ascending aorta; $\mathrm{DAO}=$ descending aorta; $\mathrm{LA}=$ left atrium; $\mathrm{LPA}=$ left pulmonary artery; $L P A W=$ left pulmonary artery wedge pressure; $L V=$ left ventricle; $\mathrm{MPA}=$ main pulmonary artery; $\mathrm{RA}=$ right atrium; $\mathrm{RPA}$; right pulmonary artery; RPAW = right pulmonary artery wedge pressure; $\mathrm{RV}=$ right ventricle.

giography (Figure 1A, Figure 2A and 2B). A trans-septal approach was utilized to access the left atrium. Using a Cobra C1 catheter, a 0.018" floppy wire was advanced from the venous sheath through the PVL and into the ascending aorta, where it was snared by a B-Braun multisnare introduced from the arterial sheath. This in effect created a veno-arterial loop of wire from the right femoral vein, across the atrial septum, through the leak, into the left ventricle, across the aortic valve, and into the descending aorta to the right femoral artery (Figure 1B). Using a 4-F Mullins sheath advanced transvenously across the PVL, a $4 \mathrm{~mm} \times 6 \mathrm{~mm}$ Amplatzer Vascular Plug II was then positioned anterogradely and was successfully deployed within the tract. Placement of the vascular plug was assisted by TEE. No significant residual leak into the left atrium was visualized by angiography or TEE (Figure $1 \mathrm{C}$, Figure $2 \mathrm{C}$ ). Post-intervention, both the left atrial pressure and mitral valve peak gradients decreased. Total fluoroscopy time was $54 \mathrm{~min}$, and the procedure time was $2 \mathrm{~h}$ and $39 \mathrm{~min}$. The patient tolerated the procedure well, and there were no associated complications.

Transthoracic echocardiography on post-procedure day 1 showed no evidence of PVL, and there was no mitral valve or pulmonary vein obstruction. 

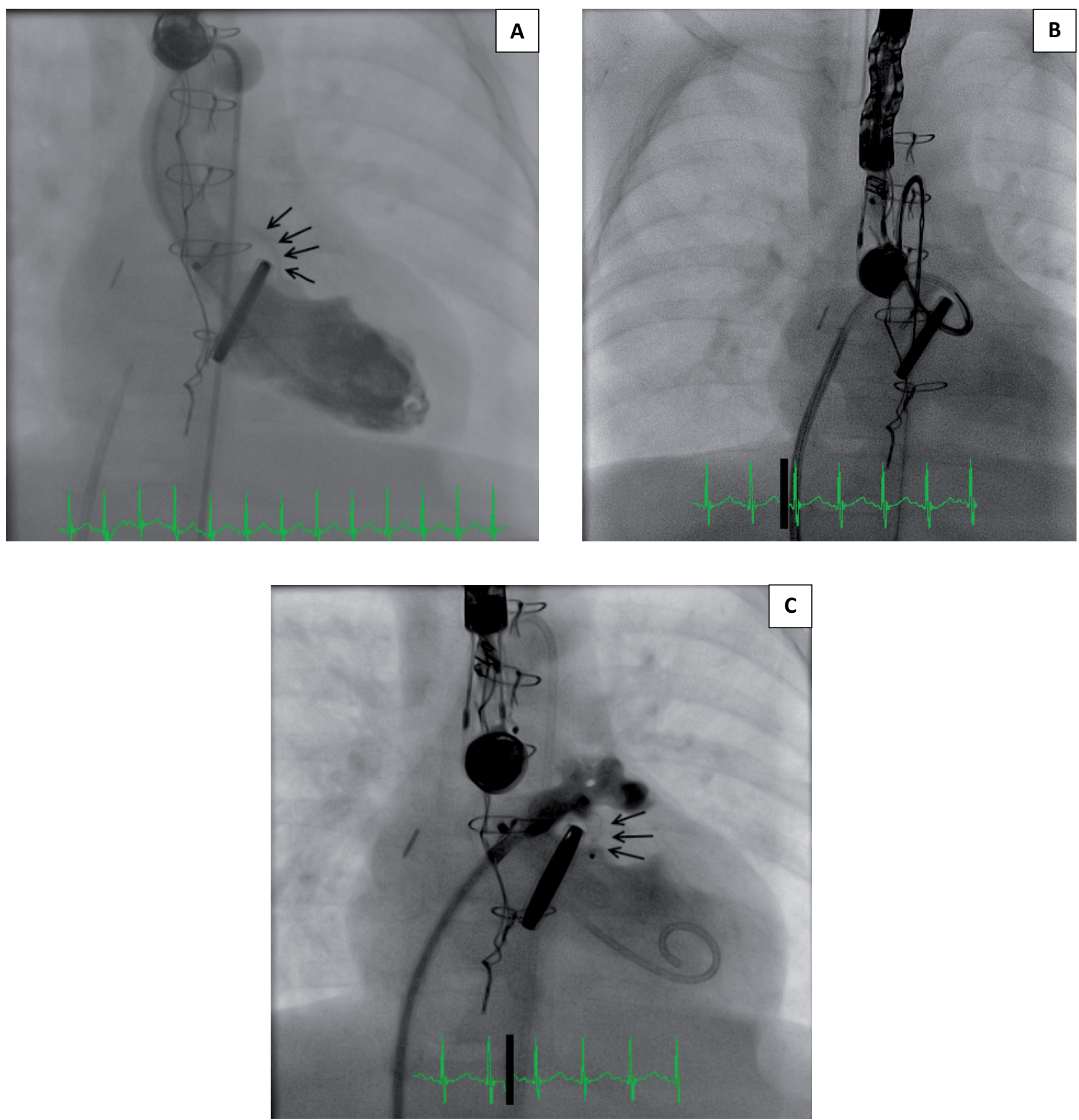

Figure 1. (Panel A) Left ventricular angiogram in the anterior posterior view showing the small lateral paravalvular leak with regurgitation into the left atrium (arrows) measuring $1.22 \mathrm{~mm}$ proximal to the LV but does become slightly larger in caliber proximal to the LA. (Panel B) Catheter course with a veno-arterial loop from the right femoral vein to the right femoral artery. (Panel C) Left atrium angiogram performed with the Amplatzer ${ }^{\circledR}$ Vascular Plug II successfully deployed in the tract with no resdiual flow (arrows).

Follow-up blood testing showed stable hemoglobin and hematocrit with resolution of hemolysis. The patient was discharged home on post-procedure day 8 after his warfarin was restarted and his international normalized ratio was therapeutic. He was seen in follow-up by his primary cardiologist 2 weeks 

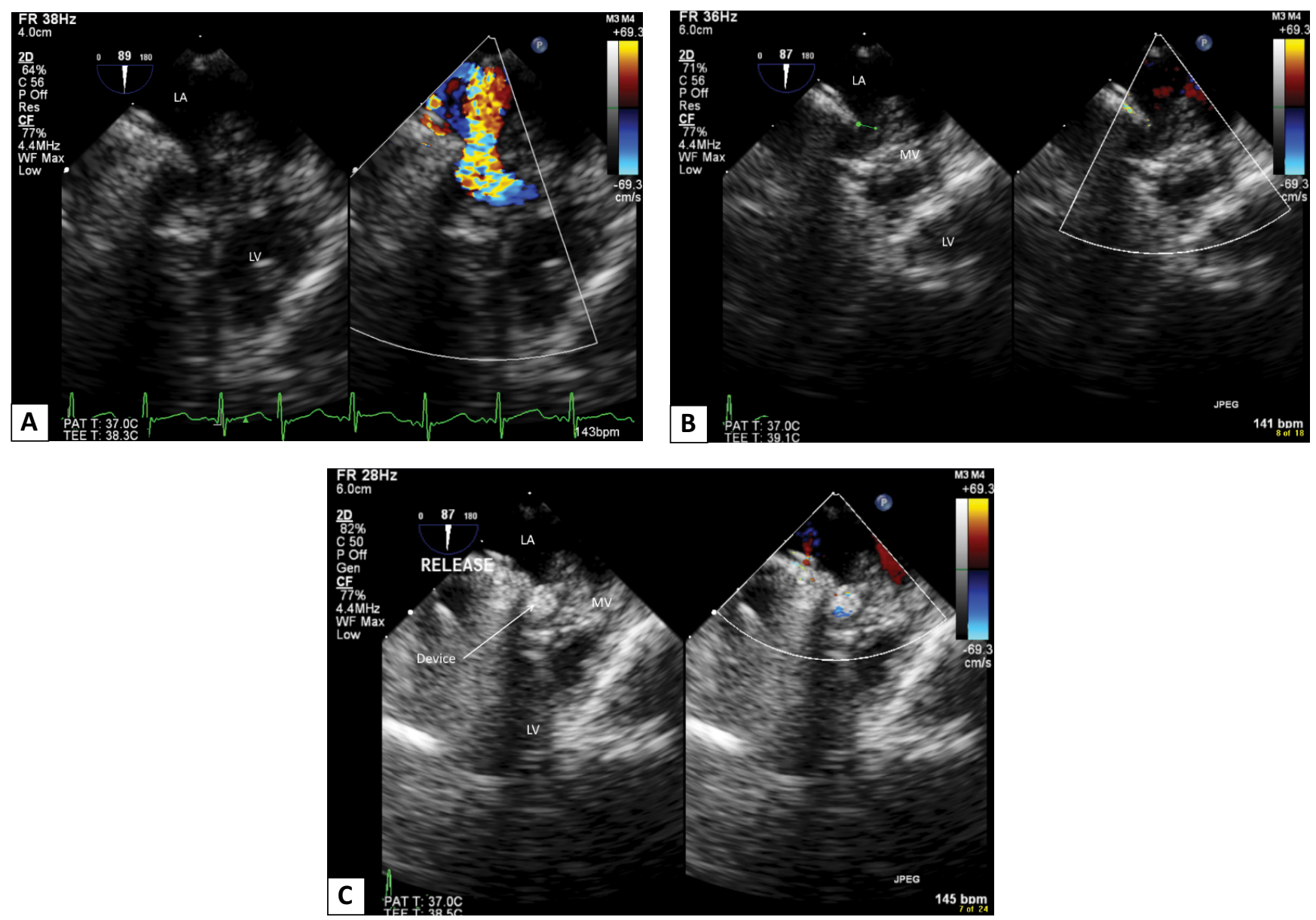

Figure 2. (Panel A) Transesophageal echocardiography showing a small-to-moderate prosthetic mitral paravalvular leak (Panel B) The defect measures $2.7 \mathrm{~mm}$. (PanelC) Status post vascular plug placement with complete resoulution of the paravalvular leak; the left atrial disk does not protrude into the left atrium. LA, left atrium; LV, left ventricle; MV, mitral valve.

post-procedure, and other than a short admission for a viral upper respiratory tract infection, he was doing well with no evidence of residual leak upon repeat imaging. At his most recent follow-up 27 months after closure of the PVL, there remained no evidence of a residual leak by transthoracic echocardiography and no recurrence of hemolysis.

\section{Discussion}

Although its true incidence in pediatric patients is not fully known, prosthetic PVL is a known complication in patients undergoing prosthetic aortic or mitral valve replacement. When significant, the gold standard therapy remains surgical closure; however, this approach is associated with higher risk, especially in young patients, which can be mitigated by transcath- eter intervention. Localizing the position of the leak in relation to the valve ring and surrounding structures can be difficult using transthoracic imaging, but utilizing peri-procedural TEE is valuable for identifying PVL anatomy and optimizing device position prior to deployment [4]. Three-dimensional TEE is another useful imaging modality in visualizing PVLs; however, its utility in smaller patients is limited due to the need for larger TEE probes.

Depending on the location of the PVL, different catheter approaches may need to be employed. Mitral valve leaks are more challenging to close because, depending on their position along the annular ring, they may be close to the atrial septum, thus producing an angle that is difficult to navigate, or left ventricular structures such as trabeculae, papillary muscles, or chordae may obstruct 


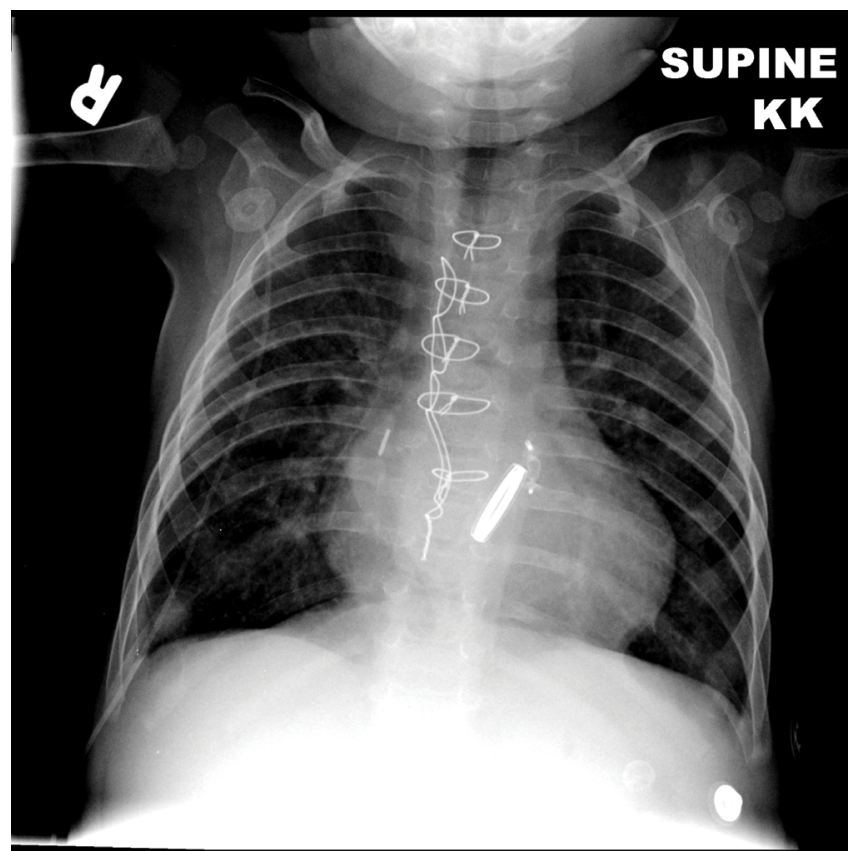

Figure 3. Post-procedure chest radiograph depicting relationship of vascular plug to the mechanical mitral valve ring.

advancing catheters and/or devices [2]. Creating a veno-arterial loop via trans-septal puncture, as was performed in our patient, allows for stable wire positioning and permits an anterograde or retrograde approach to device closure. Although there is potential for hemodynamic instability with a venoarterial loop due to the wire stenting open the aortic valve, our patient tolerated the approach well. With a pre-shaped wire loop minimizing tension across the left ventricular outflow track, it was possible to avoid hypotension related to aortic valve distortion by the wire.

An alternative approach for mitral PVL closure would be to advance the sheath directly to the left ventricle through the PVL without creating a veno-arterial loop; however, the trade-off would be attempting to advance a comparatively large sheath into a small PVL with a short length of wire of limited stability in the left ventricle. We believe that in an infant with very short left ventricular length, it would be possible but unlikely that one could maintain adequate wire position and successfully advance the 4-F sheath into position through the small, tight leak. There would possibly be increased risk of wire perforation of the left ventricular apex given the small dimensions and limited room for wire mobilization. Conversely, this approach may be more amenable with a more sizeable PVL in a larger patient. A trans-apical approach is also feasible, with the benefit of allowing direct entry of the mitral PVL regardless of its location; this can be performed percutaneously or open [2]. Disadvantages to a percutaneous apical approach are that it is more invasive and requires additional pre-procedure planning and imaging (i.e., ultrasound, echocardiography, and/ or computed tomography) and that there is limited availability of dedicated apical closure devices domestically or internationally [5-7]. The open approach has additional limitations, such as an increased risk of apical tear, bleeding, myocardial damage, arrhythmia, coronary damage, and infection.

In terms of device selection, the size of the patient must be considered for obvious technical reasons. The Amplatzer ventricular septal defect device has been used in larger patients; however, due to our patient's size and risk of iatrogenic mitral valve or pulmonary vein obstruction, we utilized the Amplatzer Vascular Plug II. This proved to be a practical device for closure of a PVL in the United States, although other candidate devices are presently available domestically (i.e., Amplatzer Duct occluder, Amplatzer septal occlude, vascular coils) and internationally (i.e., Amplatzer Vascular Plug III and 4) $[2,8]$. Newer, dedicated PVL closure devices are also on the horizon, such as the Occlutech PVL device, but due to their size, these would be better suited for larger defects in larger patients.

Successful transcatheter PVL closure has previously been described in children, adolescents, and adults. However, our report demonstrates that successful closure of a PVL in an infant under 12 months old is technically feasible, can result in clinical improvement of sequelae attributed to $\mathrm{PVL}$, and may obviate the need for repeat surgical intervention.

\section{Conflict of Interest}

The authors have no conflict of interest relevant to this publication.

\section{Comment on this Article or Ask a Question}




\section{References}

1. Millàn X, Skaf S, Joseph L, Ruiz C, Garcia E, Smolka $G$, et al. Transcatheter reduction of paravalvular leaks: a systematic review and meta-analysis. Can J Cardiol. 2015;31:260269. DOI: 10.1016/j.cjca.2014.12.012

2. Binder RK, Webb JG. Percutaneous mitral and aortic paravalvular leak repair: indications, current application, and future directions. Curr Cardiol Resp. 2013;15:342. DOI: 10.1007/s11886-012-0342-2

3. Eleid MF, Cabalka AK, Malouf JF, Sanon $S$, Hagler DJ, Rihal CS. Techniques and outcomes for the treatment of paravalvular leak. Circ Cardiovasc Interv. 2015;8:e001945. DOI: 10.1161/CIRCINTERVENTIONS.115.001945

4. O'Rourke DJ, Palac RT, Malenka DJ, Marrin $\mathrm{CA}$, Arbuckle BE, Plehn JF. Outcome of mild periprosthetic regurgitation detected by intraoperative transesophageal echocardiography. J Am Coll Cardiol. 2001;38:163-166. PMID: 11451267

5. Ferrari E. Apical access and closure devices for transapical transcatheter heart valve procedures. Swiss Med Wkly. 2016;146:w14237. DOl: 10.4414/ smw.2016.14237

6. Goktekin O, Vatankulu MA, Tasal A, Sönmez $\mathrm{O}$, Başel H, Topuz $\mathrm{U}$, et al. Transcatheter trans-apical closure of paravalvular mitral and aortic leaks using a new device: first in man experience. Catheter Cardiovasc Interv. 2014;83:308-314. DOI: 10.1002/ ccd. 25006

7. Goktekin O, Vatankulu MA, Ozhhan $\mathrm{H}, \mathrm{Ay}$ $Y$, Ergelen M, Tasal A, et al. Early experience of percutaneous paravalvular leak closure using a novel Occlutech occluder.
Eurolntervention. $\quad 2016 ; 11: 1195-1200$. DOI: 10.4244/EIJV11I10A237

8. Calvert PA, Northridge DB, Malik IS, Shapiro L, Ludman P, Qureshi SA, et al. Percutaneous device closure of paravalvular leak: combined experience from the United Kingdom and Ireland. Circulation. 2016;124:e403. DOI: 10.1161/CIRCULATIONAHA.116.022684

Cite this article as: Nwankwo $U$, Goldsmith J, Trucco S, Kreutzer J. Transcatheter Closure of a Mitral Valve Paravalvular Leak in an Infant. Structural Heart Disease. 2017;3(5):141-146. DOI: $\quad$ https://doi.org/10.12945/j. jshd.2017.019.17 